\title{
A Strange Association Between A Rectum- Infiltrating / Metastatic Dedifferentiated Chordoma And Schmidt's Syndrome
}

\author{
Endrit Shahini, Elena Maldi and Teresa Staiano* \\ FPO-IRCCS Candiolo Cancer Institute, Italy
}

ISSN: 2637-773X

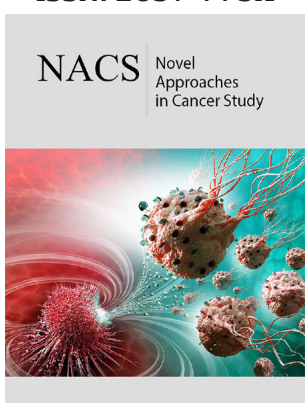

*Corresponding author: Teresa Staiano, FPO-IRCCS Candiolo Cancer Institute Candiolo, Italy

Submission: 侮 September 12, 2019

Published: 眥 November 06, 2019

Volume 3 - Issue 5

How to cite this article: Endrit Shahini, Elena Maldi, Teresa Staiano. A Strange Association Between a Rectum-Infiltrating/ Metastatic Dedifferentiated Chordoma and Schmidt's Syndrome. Nov Appro in Can Study. 3(5). NACS.000571.2019.

DOI: 10.31031/NACS.2019.03.000571

Copyright@ Teresa Staiano, This article is distributed under the terms of the Creative Commons Attribution 4.0 International License, which permits unrestricted use and redistribution provided that the original author and source are credited.

\begin{abstract}
A 57-year-old Caucasian woman, presented to our Endoscopy Unit, complaining several episodes of rectal bleeding during the last 2 weeks, associated with lower abdominal and back pain, mild weight loss and asthenia. On presentation, the patient was hemodynamically stable. Her laboratory tests showed normocytic anemia of $10 \mathrm{~g} / \mathrm{dL}$, increase of creatine chinase of $264 \mathrm{U} / \mathrm{L}$, and mild hypopotassemia (3.4 $\mathrm{mEq} / \mathrm{l}$ ). Moreover, she reported a family history for gastric cancer (father), and she was surveilled by our Oncological center for a recurrent neoplastic disease and for a systemic autoimmune disease, for many years stable under treatment with cycles of radiotherapy, imatinib mesylate, steroids, levothyroxine, and semestral zoledronic acid. After she underwent flexible sigmoidoscopy, a bulky ulcerated rectal mass, of suspected extraparietal origin, was revealed in (Figure 1) and (Figure 2). Multiple random biopsies were obtained from the normal mucosa as well as separately from the ulcers.
\end{abstract}

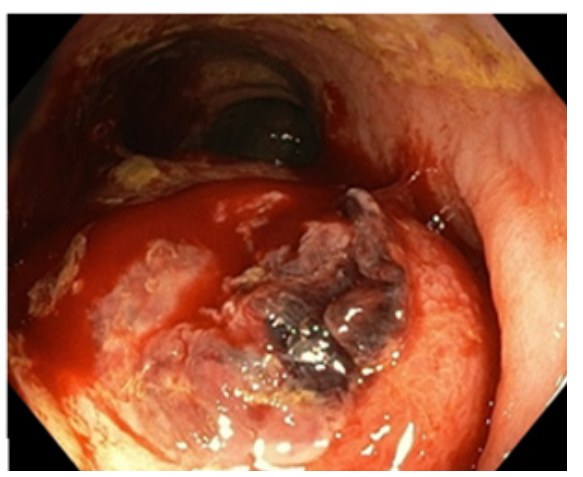

Figure 1: Endoscopic appearance of the ulcerated rectal mass, before the random biopsies performed from the normal mucosa

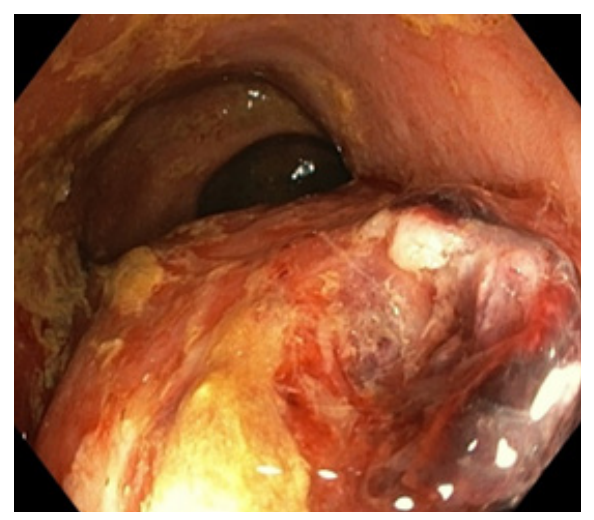

Figure 2: Endoscopic appearance of the ulcerated rectal mass, after the random biopsies performed from the normal mucosa 


\section{What is the most likely diagnosis?}

Answer to: Image 3 - A Strange Association Between a RectumInfiltrating/Metastatic Dedifferentiated Chordoma and Schmidt's Syndrome.

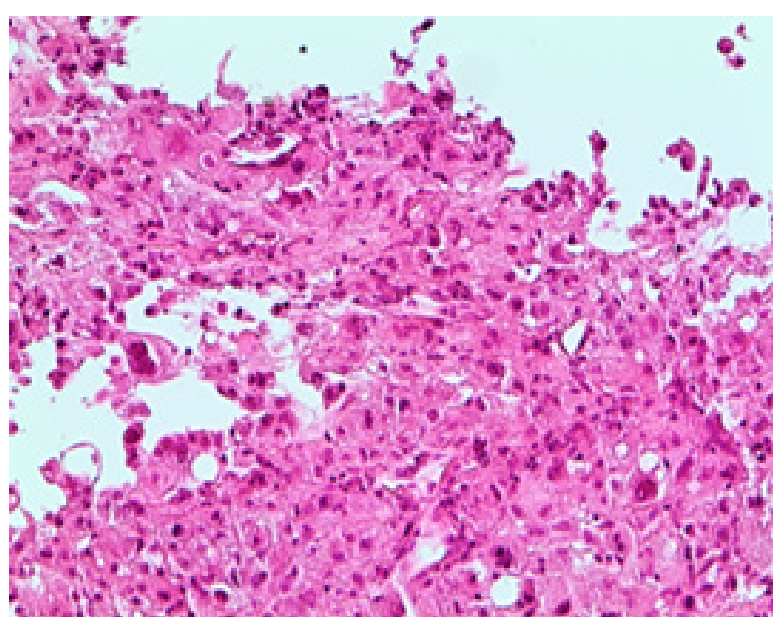

Figure 3: Rectal infiltrating chordoma on histology (H\&E stain, 20x).

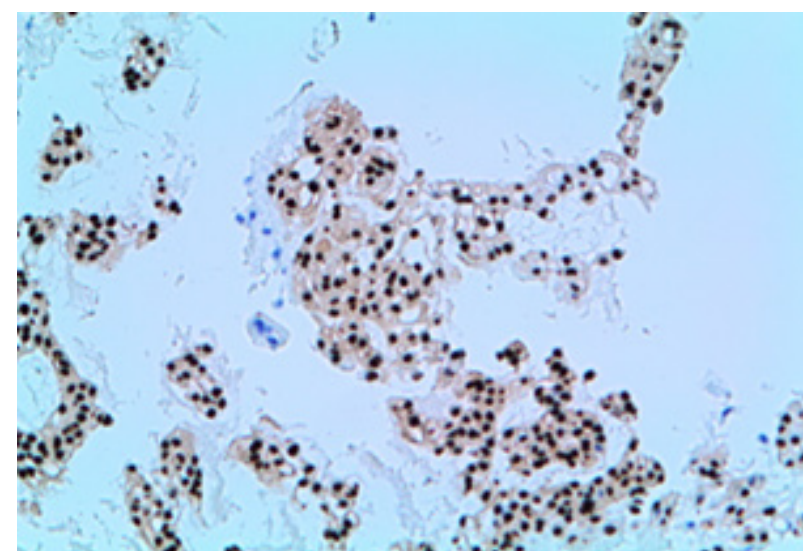

Figure 4: On immunochemistry Anti-Pancitocheratin antibody (AE1/AE3), S100, EMA, and vimentin were intensely and diffusely positive; anti-CDX2 antibody showed a weak/focal positivity (10x).

Histological specimens obtained from endoscopic biopsies showed clusters of medium/large-sized epithelioid cells, with abundant clear cytoplasm and round nuclei, suggestive of a pleomorphic mesenchymal neoplasia (Figure 3, H\&E stain, 20x). This finding was further confirmed by immunohistochemistry studies with Brachyury antibody, which showed a strong and homogeneous nuclear positivity (Figure 4). A diagnosis of dedifferentiated chordoma was finally endorsed. At the age of 43-years, the patient underwent en-bloc sacrectomy for a large sacral mass $(90 \times 60 \times 40 \mathrm{~mm})$. A postoperative diagnosis of dedifferentiated chordoma, was gained and she underwent pelvic radiotherapy (33 cycles, DFT=59.4Gy) [1,2]. Concurrently, she started steroid therapy when Schmidt's Syndrome (autoimmune polyendocrine syndrome type 2) was diagnosed. When 5 years later disease stability was achieved, on computed tomographic (CT)/ Positron Emission Tomography (PET) investigations was shown a sacral recurrence of a bulky lesion $(60 \times 35 \mathrm{~mm})$. After imatinib mesylate therapy $(600 \mathrm{mg} /$ day $)$ was added, reaching disease stability within 15 months, the pelvic mass was surgically resected. Two years later, another local disease recurrence with "de-novo" lung metastasis [3], were treated with 10 months Cisplatin cycles (30mg/mq, $-25 \%$ ), additionally to pelvic radiotherapy (5 cycles, DFT=20Gy), and 6 months later imatinib mesylate was steadily used as maintenance therapy (300-600mg/daily).

Despite initial local/systemic disease progression, within 43 months of well-tolerated therapy, the disease recurrences were considered dimensionally unchanged. When an ulcerated rectal mass was diagnosed on endoscopic assessment after the onset of rectal bleeding episodes, the next abdomen/pelvic MRI and CT scan imaging, revealed an increase of the pelvic mass $(77 \mathrm{~mm})$, which surrounded the back wall of the distal rectum, compressing and infiltrating it longitudinally for $50 \mathrm{~mm}$, with further infiltration of the posterior wall of the vagina. On the next 4 months our patient was conservatively managed (laxatives and iron replacement therapy), and rectal bleeding improved 2 weeks later without any recurrences, whereas tranexamic acid was used for treating few episodes of vaginal blood losses. As suggested by literature, a combination of surgical resection with adjuvant radiotherapy allows a good overall survival, local and distant relapse free survival in these patients, whereas imatinib is suggested for their nonsurgical management.1-3 Currently, there are no data on the most appropriate endoscopic treatment in cases of rectal bleeding due to the local advanced disease progression. New studies are warranted on this topic.

\section{Author contributions}

Shahini E collected data of the patient and drafted the manuscript; Maldi E performed the histological analysis of endoscopic biopsies; Staiano T provided content expertise; all the authors reviewed and approved the final draft.

\section{Conflicts of interest}

The authors disclose no conflicts.

\section{References}

1. van Wulfften, Tromp I, Ferreira A, Fiore A, Bramer JAM, et al. (2019) Sacral chordoma: A clinical review of 101 cases with 30-year experience in a single institution. Spine J 19(5): 869-879.

2. Catton C, O'Sullivan B, Bell R, Laperriere N, Cummings B, et al. (1996) Chordoma: Long-term follow-up after radical photon irradiation. Radiother Oncol 41(1): 67-72.

3. Casali PG, Messina A, Stacchiotti S, Tamborini E, Crippa F, et al. (2004) Imatinib mesylate in chordoma. Cancer 101(9): 2086-2097.

For possible submissions Click below: 